\title{
Quantification and Sensible Correction for Energy-Loss- and Thickness-Dependent Contrast Complications in Atomic-Scale Electron Energy-Loss Spectroscopy
}

\author{
Huolin L. Xin ${ }^{1}$, Haiyan Tan ${ }^{2}$, Christian Dwyer ${ }^{3}$, and Ye Zhu ${ }^{4}$ \\ 1. Center for Functional Nanomaterials, Brookhaven National Laboratory, Upton, NY, USA \\ 2. Materials Science and Engineering Division, National Institute of Standards and Technology, \\ Gaithersburg, Maryland, USA \\ 3. Department of Physics, Arizona State University, Tempe, AZ, USA. \\ 4. Monash Centre for Electron Microscopy and Department of Materials Engineering, Monash \\ University, Victoria, Australia
}

Atomic-resolution core-loss inelastic scattering in a scanning transmission electron microscope (STEM) is a powerful tool for resolving the local elemental composition and electronic bonding states in materials. However, due to the strong interaction of the incident electrons with the material, spatially resolved inelastic scattering signals are always modified to some extent by the unavoidable elastic scattering events, even in relatively thin samples. This modification is often evident in atomic-scale elemental maps. For example, inelastic scattering signals of unexpected strengths [1], "volcano" patterns around heavy atomic columns $[2,5]$, and "negative" contrast where the atomic columns appear "dark" $[3,4]$ have all been observed experimentally. Such effects imply that an atomic-scale elemental map does not necessarily reflect the atomic-scale elemental distribution in the material. Hence in such cases electron scattering simulations are often needed to gain a reliable interpretation. However, this process becomes a paradox for the interrogation of structurally unknown specimens. Therefore, a sensible correction method that can reliably recover the underlying chemical structures is highly needed.

In this talk, we first present a systematic study of the contrast in atomic-scale elemental maps acquired using core-loss electron energy-loss spectroscopy (EELS) in the STEM. Specifically, we perform a detailed analysis of $\mathrm{Si}-\mathrm{L}$ maps of single-crystal silicon for both a wide range of energy losses and a large range of sample thicknesses. Our results show that, for moderately thin samples $(0.5 \lambda, \lambda \approx 110 \mathrm{~nm}$ for the beam energy used here), the Si-L maps exhibit practically no contrast for energy losses within the

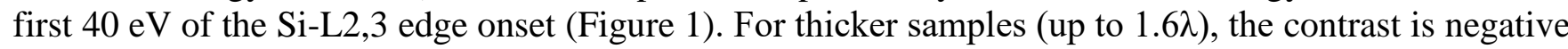
at the edge onset and evolves to become positive at higher energy losses. The energy loss at which the contrast changes from negative to positive is demonstrated to be linearly dependent on the sample thickness, a result which is also supported by our double-channeling simulations. Following our analysis of the counterintuitive contrast, we employed the method of division by quantitative incoherent bright field maps, to correct core-loss maps and restore an intuitive "positive" elemental contrast (Figure 2). This method is demonstrated to yield reliable results over the entire (large) range of energy losses and thicknesses studied here, i.e., up to energy losses of $600 \mathrm{eV}$ beyond threshold and sample thicknesses up to $1.5 \lambda$. Our detailed observations of the counterintuitive contrast in atomic-resolution elemental maps, and how to correct it, should be of great value in guiding future STEM-EELS investigations on unknown specimens. [6]

References:

[1] M. Bosman et al., Physical Review Letters 99, 086102 (2007).

[2] H. L. Xin, C. Dwyer, and D. A. Muller, Ultramicroscopy 139, 38 (2014).

[3] P. Wang et al., Physical Review Letters 101, 236102 (2008).

[4] C. Dwyer, H. L. Xin, and D. A. Muller, Physical Review B 86, 094119 (2012).

[5] Y. Zhu, A. Soukiassian, D. G. Schlom, D. A. Muller, and C. Dwyer, Appl. Phys. Lett. 103, 141908 (2013). 
[6] H.L.X. acknowledges support from Center for Functional Nanomaterials, Brookhaven National Laboratory, which is supported by the U.S. Department of Energy, Office of Basic Energy Sciences, under Contract No. DEAC02-98CH10886. This work made use of the electron microscopy facility of the Cornell Center for Materials Research (CCMR) with support from the National Science Foundation Materials Research Science and Engineering Centers (MRSEC) program (Contract No. DMR 1120296). Experimental data recording, and H.L.X. for that period, was supported by the Energy Materials Center at Cornell (emc2), an Energy Frontier Research Center funded by the U.S. Department of Energy, Office of Basic Energy Sciences, under Award No. DESC0001086. H.T. acknowledges financial support from Centre d'Elaboration de Mat'eriaux et d'Etudes Structurales, CNRS, and National Institute of Standards and Technology, USA.Y.Z. and C.D. gratefully acknowledge financial support from the Australian Research Council (Grant No. DP110104734). C.D. acknowledges support from the Ernst Ruska Centre.

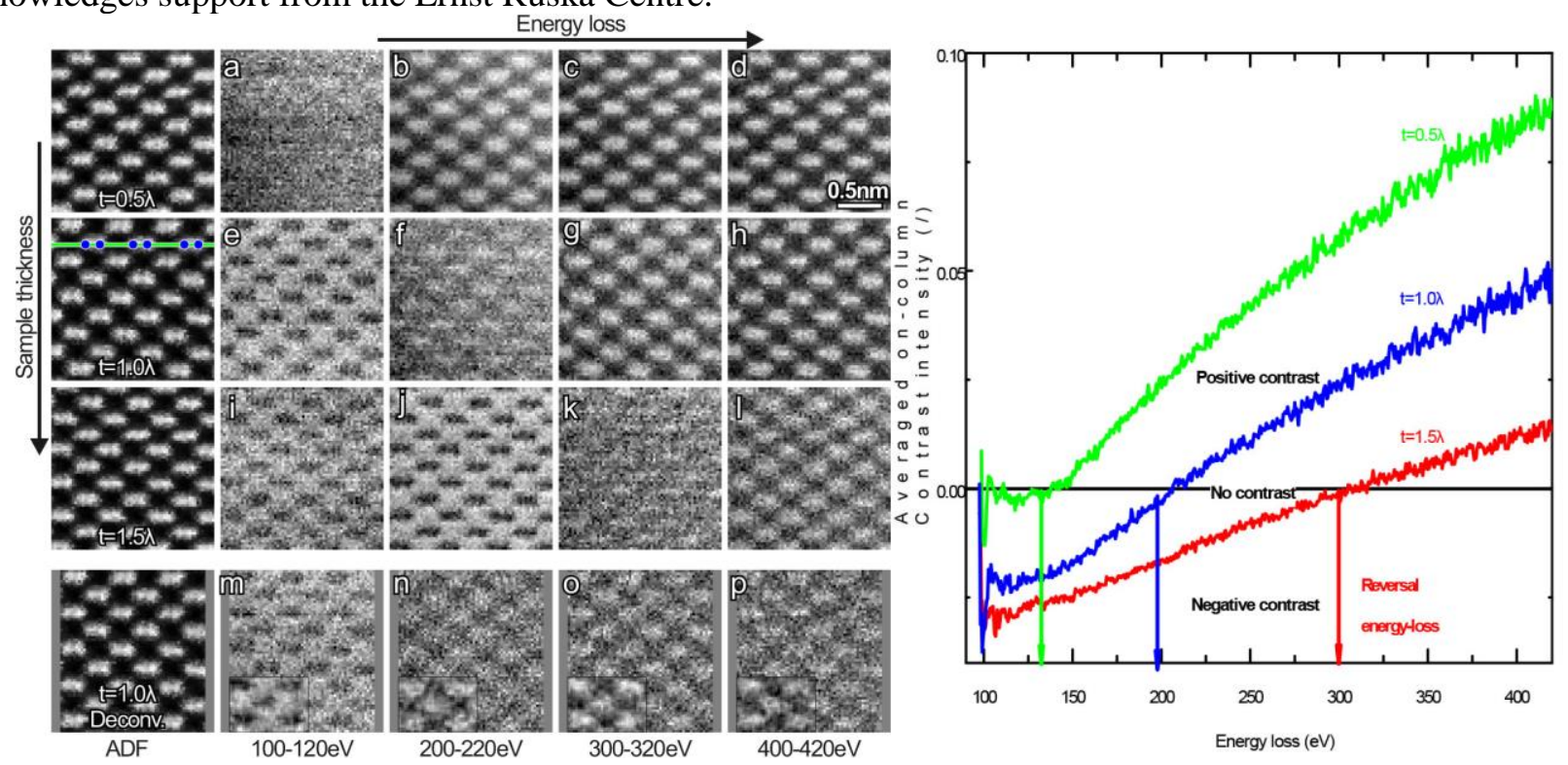

Figure 1. Atomic-resolution $\mathrm{Si}-\mathrm{L}$ maps at different energy losses and sample thicknesses (a-1). For a thickness of $1 \lambda$, maps derived after deconvolution are also shown $(m-p)$, where the insets show the results of low-pass Gaussian filtering with a FWHM of $0.04 \mathrm{~nm}$. The simultaneously acquired ADF images are shown on the left.

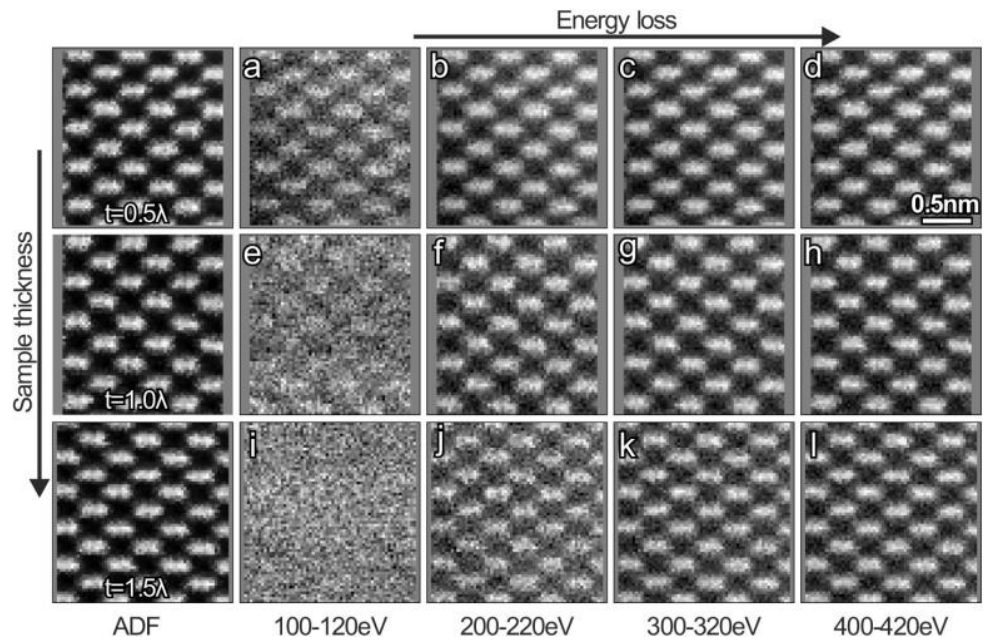

Figure 2. Corrected atomic-resolution $\mathrm{Si}-\mathrm{L}$ maps at different energy losses and sample thicknesses (a1). The simultaneously acquired ADF images are shown on the left. The maps exhibit intuitive chemical contrast in all cases, except in (i) where the map is dominated by noise. 